\section{Prevalência e fatores associados à violência entre parceiros íntimos após a revelação do diagnóstico de doenças sexualmente transmissíveis ao parceiro}

\author{
Prevalence of intimate partner violence and \\ associated factors after disclosing the diagnosis of \\ a sexually transmissible disease
}

\section{Prevalencia y factores asociados a prácticas violentas entre parejas sentimentales tras la revelación del diagnóstico de enfermedades sexualmente transmisibles}

Roumayne Fernandes Vieira Andrade Maria Alix Leite Araújo 1 Maria Inês Costa Dourado 2 Angelica Barbosa Espinosa Miranda ${ }^{3}$ Claudia Bastos da Silveira Reis 4

\begin{abstract}
Resumo
O objetivo do trabalho é investigar os fatores associados à violência por parceiro íntimo perpetrada após a revelação do diagnóstico de doenças sexualmente transmissíveis (DST), em Fortaleza, Ceará, Brasil. Estudo transversal realizado com 221 pessoas atendidas em serviços de referência para DST. Realizou-se análise multivariada com modelo de regressão logística. Praticaram algum tipo de violência por parceiro íntimo após a revelação do diagnóstico $28,1 \%$ das pessoas. Praticar violência por parceiro íntimo apresentou associação com o uso de álcool (OR = 2, 79; IC95\%: 1,256,22; $p=0,012$ ), o parceiro ter se relacionado com outra pessoa durante $o$ relacionamento atual $(O R=4,71$; IC95\%: $2,24-9,91 ; p=0,000)$, ter comet $i$ do violência anterior à DST $(O R=2,87$; IC95\%: 1,22-6,73; $p=0,015)$ e ter sofrido violência após o diagnóstico de DST (OR = 6,53; IC95\%: 3,06-13,93; $p=0,000$ ). A violência por parceiro íntimo após a revelação do diagnóstico de DST sinaliza que profissionais que atendem esta demanda devem identificar as dificuldades enfrentadas pelo paciente ao revelar o diagnóstico ao parceiro.
\end{abstract}

Doenças Sexualmente Transmissíveis; Violência por Parceiro Íntimo; Diagnóstico; Violência

\footnotetext{
1 Universidade de Fortaleza, Fortaleza, Brasil.

2 Instituto de Saúde Coletiva Universidade Federal da Bahia, Salvador, Brasil. 3 Universidade Federal do Espírito Santo, Vitória, Brasil. ${ }^{4}$ Hospital Distrital Gonzaga Mota de Messejana, Fortaleza, Brasil.
}

\author{
Correspondência \\ R. F. V. Andrade \\ Universidade de Fortaleza. \\ Av. Washington Soares 1321, \\ Fortaleza, CE \\ 60811-905, Brasil. \\ roumaynefv@hotmail.com
}




\section{Introdução}

A violência é um fenômeno que tem aumentado muito nos últimos anos e um dos principais problemas de saúde pública na atualidade. O seu enfrentamento é complexo e necessita de ações intersetoriais. A violência perpetrada por parceiro íntimo refere-se a "qualquer comportamento dentro de uma relação íntima que cause dano físico, psíquico ou sexual a qualquer um dos membros da relação" 1 (p. 89).

Alguns estudos internacionais 2,3 indicam que a violência por parceiro íntimo é frequente em pessoas com doenças sexualmente transmissíveis (DST). No Brasil, a associação entre episódios de violência e a ocorrência de DST mostra que vítimas de violência se encontram mais vulneráveis a contrair uma DST 4,5. Uma pesquisa realizada em clínicas de planejamento familiar na Califórnia (Estados Unidos) encontrou que mulheres expostas à violência por parceiro íntimo relataram medo de comunicar o diagnóstico da DST ao parceiro sexual 6 .

O foco predominante da maioria dos estudos sobre violência por parceiro íntimo coloca a mulher como vítima e o parceiro masculino como agressor, desconsiderando que as mulheres também podem assumir o papel de agressoras 7. A violência por parceiro íntimo quando associada à DST pode tornar a situação ainda mais complexa.

Pessoas que vivenciam situações de violência, por diversas razões, tendem a ficar mais vulneráveis ao adoecimento ${ }^{8,9}$, gerando demandas de saúde que envolvem necessidades de cuidados físicos, emocionais e psicológicos. Muitas vezes, essas situações não são identificadas pelos serviços de saúde por falta de preparo dos profissionais de saúde no manejo dos casos, e a falta de reconhecimento da situação como um problema de saúde 10,11.

Este artigo tem como objetivo investigar a prevalência e os fatores associados à perpetração de violência por parceiro íntimo em portadores de doenças sexualmente transmissíveis, após a revelação do diagnóstico ao parceiro na cidade de Fortaleza, Ceará, Brasil.

\section{Metodologia}

Estudo de corte transversal que analisou a prevalência de violência por parceiro íntimo após a revelação do diagnóstico de DST. violência por parceiro íntimo foi definida como qualquer ato intencional em uma relação íntima que cause dano físico, psíquico ou sexual aos membros da relação. Esse comportamento inclui agressões físicas, violência psicológica, relações sexuais forçadas e outras formas de coação sexual, além de diversos comportamentos dominantes, como isolar uma pessoa da família ou amigos, vigiar seus movimentos ou restringir seu direito à informação ou à assistência 1. Considerou-se como parceiro íntimo o esposo(a), namorado(a), noivo(a) ou qualquer outra pessoa com quem se desenvolva uma relação íntimo-afetiva, independentemente do tempo da relação.

O estudo foi realizado nos três serviços públicos de referência para tratamento de pessoas com DST da cidade de Fortaleza. Esses serviços atendem demanda espontânea ou encaminhada da atenção primária e de outros serviços de saúde.

Política e administrativamente, Fortaleza está dividida em seis Secretarias Regionais (SR), regiões administrativas que na área da saúde trabalham de forma articulada, desenvolvendo ações para alcançar as metas estabelecidas pela Secretaria Municipal de Saúde (SMS; http://www. fortaleza.ce.gov.br, acessado em 23/Out/2011) para cada grupo populacional, prestando serviços por meio de uma rede hierarquizada e de proteção social.

Participaram do estudo pessoas com diagnóstico sindrômico e/ou etiológico de DST, que se encontravam em atendimento ambulatorial nos referidos serviços. Trabalhou-se com uma amostra de 221 pacientes, calculada com base no total de 8.966 atendimentos de DST ocorridos no ano de 2011, frequência esperada de $18 \%$ de violência entre parceiros íntimos com DST 2, erro amostral de $5 \%$ e intervalo de $95 \%$ de confiança (IC95\%). As informações referentes ao quantitativo de atendimento foram fornecidas pelos Serviços de Arquivo Médico e Estatístico (SAME) de cada uma das unidades participantes da pesquisa. Retornos de pacientes ao serviço para avaliação não foram considerados atendimentos.

Os dados foram coletados entre os meses de março e setembro de 2012, por meio de um questionário aplicado face a face aos participantes do estudo, para evitar barreiras educacionais. Foram convidadas a participar da pesquisa todas as pessoas que aguardavam atendimento nos ambulatórios dos serviços de referência e que apresentavam diagnóstico etiológico ou sindrômico de úlcera genital, corrimento uretral, corrimento vaginal/cervicite, verruga genital e dor pélvica 12 .

No questionário, constavam variáveis sociodemográficas (sexo, idade, escolaridade, renda pessoal e familiar); comportamentais (tipo de DST, número de parceiros sexuais, orientação sexual, se manteve relação sexual com outra pessoa, uso de álcool e drogas, e uso de preservativo); institucionais (abordagem da violência pelo ser- 
viço de saúde); e referentes à violência praticada antes e após o diagnóstico da DST (natureza da violência, intensidade da sua ocorrência). A variável dependente foi praticar violência por parceiro íntimo após a revelação do diagnóstico de DST.

As questões relacionadas à violência por parceiro íntimo foram elaboradas utilizando-se como referência o questionário padronizado e validado do Estudo Multipaíses sobre a Saúde da Mulher e Violência Doméstica da Organização Mundial da Saúde (OMS) e validado no Brasil 1,13. Foi considerada prática de violência por parceiro íntimo quando o entrevistado respondeu afirmativamente a alguma das perguntas sobre violência.

Realizou-se um teste piloto com o objetivo de aperfeiçoar o questionário, proporcionar maior entendimento das perguntas, evitar dúvidas e possibilitar a realização dos devidos ajustes 14 .

Foram incluídos os portadores de DST que tinham revelado o diagnóstico ao parceiro sexual e recebido pelos menos duas consultas na unidade para que houvesse tempo hábil de revelar o diagnóstico. Foram excluídas as pessoas que viviam com HIV/AIDS porque o objetivo do estudo é mostrar a repercussão da violência por parceiro íntimo entre portadores de outras DST; pacientes que se encontravam em primeira consulta; pessoas com distúrbios mentais e que não tinham parceiro sexual na ocasião do diagnóstico.

Os dados foram digitados e armazenados utilizando-se o pacote estatístico IBM SPSS, versão 19.0 (IBM Corp., Armonk, Estados Unidos). Foi realizada uma análise descritiva usando-se a distribuição de frequências para as variáveis categóricas, cálculo de média e desvio padrão para as variáveis numéricas. Para a análise bivariada, foi aplicado o teste qui-quadrado de Pearson e o teste exato de Fisher, quando pertinente, para analisar associações estatísticas entre as variáveis categóricas, estabelecendo-se um nível de significância de 5\% e IC95\%.

A análise multivariada foi realizada pelo pacote estatístico Stata, versão 11.0 (StataCorp LP, College Station, Estados Unidos), por meio do modelo de regressão logística. Para a análise ajustada, permaneceram as variáveis que obtiveram o valor de $\mathrm{p}<0,05$. Todas as covariáveis foram inseridas simultaneamente no modelo múltiplo de regressão. Como medida de efeito, usou-se a razão de chances (OR), com IC95\%.

Esta pesquisa faz parte de um estudo intitulado violência por parceiro íntimo após diagnóstico de DST em Fortaleza, que recebeu aprovação do Comitê de Ética em Pesquisa da Universidade de Fortaleza (UNIFOR), com parecer no 437/2011, e do Hospital Universitário Walter Cantídio, com parecer no 043.06.12.
Foi conferido aos participantes o direito de decidir pela participação. A identidade e os dados dos participantes foram mantidos em sigilo e todos assinaram termo de consentimento livre e esclarecido.

\section{Resultados}

Participaram do estudo 221 pessoas com DST (59,7\% mulheres e 40,7\% homens). Praticaram algum tipo de violência por parceiro íntimo após a revelação do diagnóstico, $63(28,1 \%)$ pessoas (98,4\% psicológica, $25,4 \%$ física e $1,6 \%$ sexual). Foram diagnosticados com verruga genital $68,3 \%$, sífilis $18,6 \%$, herpes genital $8,1 \%$, gonorreia $3,2 \%$, tricomoníase $0,9 \%$, doença inflamatória pélvica (DIP) e donovanose $0,5 \%$.

A idade dos entrevistados variou de 12 a 80 anos (média $=30$, mediana $=29$, moda $=23$ e desvio padrão $=11,6)$. Tinham até 19 anos, 19,5\%. A escolaridade foi superior a cinco anos de estudos para 195 (88,2\%) pessoas, e 142 (64,3\%) exerciam alguma atividade remunerada. Declararam-se pardos ou pretos, 178 (80,5\%). Eram casados ou viviam em união consensual, $153(69,2 \%)$ e 68 (30,8\%) eram solteiros(as), mas tinham companheiro(a). Referiram mais de um parceiro sexual nos últimos três meses anteriores à entrevista, 49 (22,2\%). A renda pessoal foi de até um salário mínimo para 141 (63,8\%) e 72 pessoas (32,6\%) referiram não ter nenhuma renda. A renda familiar era superior a um salário mínimo para 167 (75,6\%) entrevistados.

A grande maioria, 192 (86,9\%), iniciou a vida sexual com menos de 19 anos (média $=16,3$, mediana $=16$, moda $=15$ e desvio padrão $=3,3$ ) e 199 (90\%) tiveram mais de um parceiro sexual na vida (média $=17,2$, mediana $=5,0$, oda $=3,0$, desvio padrão = 79,0). Referiram ter ciúmes do(a) parceiro(a), 165 (74,7\%).

A Tabela 1 traz uma análise bivariada das variáveis sociodemográficas e prática de violência por parceiro íntimo após o diagnóstico de DST. Praticaram violência pessoas com mais de 19 anos (30,3\%), com menos de cinco anos de estudos $(34,6 \%)$, que se declararam pardos ou pretos (30,3\%), que estavam casados ou em união consensual $(19,4 \%)$, que não estudavam $(28,6 \%)$ e que tinham renda superior a um salário mínimo (30\%). Ser do sexo feminino foi a única variável sociodemográfica que apresentou significância estatística para praticar violência por parceiro íntimo ( $\mathrm{p}=0,004)$.

Quando analisadas as variáveis comportamentais das pessoas com DST que praticaram violência por parceiro íntimo após o diagnóstico, encontrou-se associação estatisticamente significante para o início das atividades sexuais com 
Variáveis sociodemográficas associadas à prática de violência por parceiro íntimo após diagnóstico de doença sexualmente transmissível. Fortaleza, Ceará, Brasil, 2012.

\begin{tabular}{|c|c|c|c|c|c|}
\hline \multirow[t]{3}{*}{ Variáveis } & \multicolumn{4}{|c|}{ Praticou violência } & \multirow[t]{3}{*}{ Valor de $p$} \\
\hline & \multicolumn{2}{|c|}{ Sim } & \multicolumn{2}{|c|}{ Não } & \\
\hline & $\mathbf{n}$ & $\%$ & $\mathbf{n}$ & $\%$ & \\
\hline Sexo & & & & & 0,004 \\
\hline Feminino & 47 & 35,6 & 85 & 64,4 & \\
\hline Masculino & 16 & 18,0 & 73 & 82,0 & \\
\hline Faixa etária (anos) & & & & & 0,220 \\
\hline$\leq 19$ & 9 & 20,9 & 124 & 69,7 & \\
\hline$>19$ & 54 & 30,3 & 34 & 79,1 & \\
\hline Escolaridade (anos) & & & & & 0,463 \\
\hline$\leq 5$ & 9 & 34,6 & 17 & 65,4 & \\
\hline$>5$ & 54 & 27,7 & 141 & 72,3 & \\
\hline Cor da pele (autorreferida) & & & & & 0,220 \\
\hline Branca & 9 & 20,9 & 34 & 79,1 & \\
\hline Parda ou preta & 54 & 30,3 & 124 & 69,7 & \\
\hline Procedência & & & & & 0,662 \\
\hline Fortaleza & 53 & 29,1 & 129 & 70,9 & \\
\hline Outra localidade & 10 & 25,6 & 29 & 74,4 & \\
\hline Estado civil & & & & & 0,655 \\
\hline Casado/União consensual & 45 & 29,4 & 108 & 70,6 & \\
\hline Solteiro & 18 & 26,5 & 50 & 73,5 & \\
\hline Trabalha & & & & & 0,433 \\
\hline Sim & 43 & 30,3 & 99 & 69,7 & \\
\hline Não & 20 & 25,3 & 59 & 74,7 & \\
\hline Estuda & & & & & 0,972 \\
\hline Sim & 17 & 28,3 & 43 & 71,7 & \\
\hline Não & 46 & 28,6 & 115 & 71,4 & \\
\hline Renda pessoal (salários mínimos) & & & & & 0,711 \\
\hline$\leq 1$ & 39 & 27,7 & 102 & 72,3 & \\
\hline$>1$ & 24 & 30,0 & 59 & 74,7 & \\
\hline Total & & & & & \\
\hline
\end{tabular}

idade superior a 15 anos $(p=0,016)$, ter tido um único parceiro sexual na vida $(\mathrm{p}=0,004)$, tempo de relacionamento superior a um ano $(\mathrm{p}=0,025)$, pessoas cujo parceiro se relacionou sexualmente com outra pessoa durante o relacionamento atual ( $p=0,000)$, considerar que contraiu a DST através da relação sexual ( $p=0,002)$, pessoas que faziam uso de bebida alcoólica $(p=0,020)$ e que tinham cometido violência antes da DST ( $\mathrm{p}=$ 0,000) (Tabela 2).

A Tabela 3 apresenta uma análise multivariada não ajustada e ajustada para a prática de violência após o diagnóstico de DST. Praticar violência apresentou associação estatisticamente significativa com o uso de álcool $(\mathrm{OR}=2,79$; IC95\%: $1,25-6,22 ; p=0,012$ ), o parceiro ter se relaciona- do com outra pessoa durante o relacionamento atual (OR = 4,71; IC95\%: 2,24-9,91; $p=0,000)$, ter cometido violência anterior à DST (OR = 2,87; IC95\%: 1,22-6,73; $\mathrm{p}=0,015)$ e ter sofrido violência após o diagnóstico de DST (OR =6,53; IC95\%: 3,06-13,93; $\mathrm{p}=0$ 0,000).

\section{Discussão}

A prevalência de violência perpetrada por parceiro íntimo após o diagnóstico de DST foi de $28,5 \%$.

$\mathrm{Na}$ análise bivariada das variáveis sociodemográficas, apenas o sexo foi estatisticamente significante para a prática de violência por 
Variáveis comportamentais associadas à prática de violência por parceiro íntimo após diagnóstico de doença sexualmente transmissível (DST). Fortaleza, Ceará, Brasil, 2012.

\begin{tabular}{|c|c|c|c|c|c|}
\hline \multirow[t]{3}{*}{ Variáveis } & \multicolumn{4}{|c|}{ Praticou violência } & \multirow[t]{3}{*}{ Valor de $p$} \\
\hline & \multicolumn{2}{|c|}{ Sim } & \multicolumn{2}{|c|}{ Não } & \\
\hline & $\mathbf{n}$ & $\%$ & $\mathrm{n}$ & $\%$ & \\
\hline Orientação sexual & & & & & 0,378 \\
\hline Heterossexual & 58 & 29,4 & 139 & 70,6 & \\
\hline Homossexual & 5 & 20,8 & 19 & 79,2 & \\
\hline Início da atividade sexual (anos) & & & & & 0,016 \\
\hline$\leq 15$ & 20 & 20,4 & 78 & 79,6 & \\
\hline$>15$ & 43 & 35,2 & 79 & 64,8 & \\
\hline Número de parceiros sexuais (últimos 3 meses) & & & & & 0,927 \\
\hline 1 & 49 & 28,5 & 123 & 71,5 & \\
\hline$>1$ & 14 & 28,6 & 35 & 71,4 & \\
\hline Número de parceiros (na vida) & & & & & 0,004 \\
\hline 1 & 12 & 54,4 & 10 & 45,5 & \\
\hline$>1$ & 51 & 25,6 & 148 & 74,4 & \\
\hline Tempo de relacionamento (anos) & & & & & 0,025 \\
\hline$\leq 1$ & 10 & 17,2 & 48 & 82,8 & \\
\hline$>1$ & 53 & 32,7 & 109 & 67,3 & \\
\hline Tipo de DST & & & & & 0,329 \\
\hline Verruga genital & 40 & 26,5 & 111 & 73,5 & \\
\hline Outra & 23 & 32,9 & 47 & 67,1 & \\
\hline Parceiro teve outros parceiros durante o relacionamento atual & & & & & 0,000 \\
\hline Sim & 42 & 48,8 & 44 & 51,2 & \\
\hline Não & 21 & 15,6 & 114 & 84,4 & \\
\hline Teve outros parceiros durante o relacionamento atual & & & & & 0,531 \\
\hline Sim & 22 & 31,4 & 48 & 68,6 & \\
\hline Não & 41 & 27,3 & 109 & 72,7 & \\
\hline Considera ter adquirido a DST na relação sexual & & & & & 0,716 \\
\hline Sim & 58 & 28,9 & 143 & 71,1 & \\
\hline Não & 5 & 25,0 & 15 & 75,0 & \\
\hline Usa álcool & & & & & 0,020 \\
\hline $\operatorname{Sim}$ & 38 & 35,8 & 68 & 64,2 & \\
\hline Não & 25 & 21,7 & 90 & 78,3 & \\
\hline Usa droga & & & & & 0,542 \\
\hline Sim & 2 & 20,0 & 8 & 80,0 & \\
\hline Não & 61 & 28,9 & 150 & 71,1 & \\
\hline Praticou violência antes da DST & & & & & 0,000 \\
\hline Sim & 24 & 52,2 & 22 & 47,8 & \\
\hline Não & 39 & 22,3 & 136 & 77,7 & \\
\hline Teve receio de revelar o diagnóstico ao parceiro & & & & & 0,631 \\
\hline Sim & 20 & 30,8 & 45 & 69,2 & \\
\hline Não & 43 & 27,6 & 113 & 72,4 & \\
\hline
\end{tabular}

parceiro íntimo ( $\mathrm{p}=0,004)$, não se mantendo na análise multivariada ( $\mathrm{p}=0,095)$. Um estudo de base populacional, realizado com mulheres e homens suecos, encontrou que mais mulhe- res do que homens relataram praticar violência por parceiro íntimo ${ }^{7}$. As mulheres referiram se envolver mais que os homens em episódios de perpetração da violência por parceiro íntimo, 
Odds ratio (OR) da associação entre a prática de violência por parceiro íntimo após a revelação do diagnóstico de doença sexualmente transmissível (DST) com as variáveis comportamentais. Fortaleza, Ceará, Brasil, 2012.

\begin{tabular}{|c|c|c|c|c|c|}
\hline & $n / N$ & $\%$ & OR & IC95\% & Valor de $p$ \\
\hline Uso de álcool pelo portador da DST & & & 2,79 & $1,25-6,22$ & 0,012 \\
\hline Sim & $38 / 106$ & 35,8 & & & \\
\hline Não & $25 / 115$ & 21,7 & & & \\
\hline Número de parceiros sexuais na vida & & & 0,11 & $0,03-0,36$ & 0,000 \\
\hline$>1$ & $51 / 199$ & 80,9 & & & \\
\hline 1 & $12 / 22$ & 19,5 & & & \\
\hline Parceiro teve outro parceiro sexual & & & 4,71 & $2,24-9,91$ & 0,000 \\
\hline Sim & $42 / 86$ & 48,8 & & & \\
\hline Não & $21 / 135$ & 15,6 & & & \\
\hline Foi violento antes da DST & & & 2,87 & $1,22-6,73$ & 0,015 \\
\hline Sim & $24 / 46$ & 52,1 & & & \\
\hline Não & $39 / 175$ & 22,3 & & & \\
\hline Sofreu violência após a DST & & & 6,53 & $3,06-13,93$ & 0,000 \\
\hline Sim & $38 / 67$ & 56,7 & & & \\
\hline Não & $25 / 154$ & 16,2 & & & \\
\hline
\end{tabular}

IC95\%: intervalo de $95 \%$ de confiança.

situação também encontrada em uma pesquisa brasileira que estimou a prevalência de violência por parceiro íntimo e o consumo de álcool durante os eventos violentos 15 .

No Brasil, o Ministério da Saúde implantou programas específicos de atenção à mulher em situação de violência, o Poder Legislativo criou leis de punição para os agressores e foram implantadas delegacias específicas para este tipo de atendimento. Atualmente, já se dispõe de vários mecanismos para tentar coibir a violência doméstica e familiar. Dentre essas estratégias, pode-se destacar a Lei Maria da Penha 16, os Juizados de Violência Doméstica e Familiar, as delegacias para atendimento à mulher que sofre violência, além de casas de abrigo.

A bebida alcoólica contribui para as práticas de violência por parceiro íntimo 17 e aumenta a ocorrência e gravidade da violência doméstica 18,19 . Neste estudo, encontrou-se que pessoas que fazem uso de álcool têm duas vezes mais chances de praticar violência por parceiro íntimo após a revelação do diagnóstico de DST. O álcool reduz o autocontrole, afeta o funcionamento cognitivo e físico 20 , o que pode reduzir a capacidade do indivíduo para a resolução dos conflitos do relacionamento de forma não violenta. O consumo de álcool aumenta a ocorrência e gravidade da violência doméstica 18 .

Pessoas que vivenciam situação de violência estão mais propensas a contrair DST 2,3,4,5. Um estudo realizado com homens heterossexuais em Nova York (Estados Unidos), que avaliou a relação entre perpetração de violência por parceiro íntimo e uso inconsistente do preservativo, encontrou que homens que perpetraram violência física contra suas parceiras tinham metade da probabilidade de relatar o uso consistente do preservativo, em comparação com os homens que não praticaram violência 21 . A violência dificulta a inserção de práticas preventivas das DST, pois pessoas que vivenciam a violência por parceiro íntimo têm receio de solicitar o uso do preservativo nas relações sexuais 22 .

As questões que envolvem a violência por parceiro íntimo são multifatoriais e de difícil solução. Em se tratando de pessoas com o diagnóstico de DST, a possibilidade da infidelidade como um dos motivos para o surgimento da DST pode ser uma das causas da violência por parceiro íntimo 23 . As chances de praticar a violência contra o parceiro íntimo foram quatro vezes maiores quando o paciente tinha conhecimento que seu parceiro havia se relacionado sexualmente com outra pessoa durante o relacionamento atual.

A infidelidade, especialmente quando praticada pela mulher, é pouco tolerada na sociedade e o diagnóstico de uma DST em algum dos membros da relação pode ser considerado como a comprovação da traição. Este trabalho mostrou que o diagnóstico de DST desencadeia atos violentos na relação, e o profissional de saúde 
deve considerar este aspecto durante o aconselhamento e especialmente a convocação dos parceiros sexuais. Esse pode ser um motivo importante da dificuldade em tratar os parceiros sexuais de portadores de DST 6. Por esse motivo, o aconselhamento deve discutir esse aspecto da violência, visando a contribuir para evitar o desencadeamento dessas reações entre parceiros sexuais. Diante da suspeita de relações extraconjugais por parte do parceiro, mulheres podem tornar-se abusivas por causa do ciúme 24 .

A maioria das pesquisas sobre violência tem sido realizada com mulheres e as consideram vítimas de violência 25 . No entanto, em contraste com outras formas de violência interpessoal, a violência por parceiro íntimo é muitas vezes perpetrada também por mulheres 7,15.

A reciprocidade de atos violentos é comum em relações íntimas 26,27, situação que parece ter ocorrido também entre as pessoas com DST envolvidas neste estudo, que encontrou que aquelas que praticaram violência após a revelação da DST tinha seis vezes mais chances de sofrer violência por parceiro íntimo.

Um estudo realizado com adultos jovens norte-americanos, encontrou que em cerca da metade das relações violentas os atos eram recíprocos, que as mulheres estão em maior risco para sofrer violência mais grave e que estas foram as autoras da violência em mais de $70 \%$ dos casos 28 .

O aconselhamento é uma importante estratégia para a notificação do parceiro e adesão ao tratamento. Essa estratégia tem um impacto significantemente positivo no encaminhamento para o tratamento de parceiros sexuais de pessoas com DST 28. Apesar de mais ênfase ser dada ao HIV 29, a comunicação ao parceiro é fundamental, independentemente da DST, pois proporciona aos parceiros expostos a oportunidade de avaliação e tratamento médico, limitando a propagação da infecção.

A perpetração está relacionada a viver com o parceiro 27 . Nesta pesquisa, praticar violência por parceiro íntimo foi mais frequente entre as pessoas casadas ou em união consensual, e a perpetração da violência esteve associada a pessoas com mais de um ano de relacionamento, o que pode aumentar as oportunidades de violência $e$ relações de tensão entre os membros da relação.

A perpetração da violência por parceiro íntimo após o diagnóstico de DST esteve associado à prática da violência anteriormente à DST, confirmando a natureza repetitiva da violência por parceiro íntimo. Esse tipo de violência é de difícil rompimento, pois envolve questões afetivas, econômicas e familiares ${ }^{30}$. Mulheres vítimas de violência física chegam a se separar dos seus companheiros por causa das agressões, mas cerca de dois terços voltam ao convívio com o agressor 31 .

Os homens e mulheres que demandaram atendimento em clínicas de DST apresentaram uma alta prevalência de verruga genital; neste estudo a infecção pelo papiloma vírus humano (HPV) atingiu 68,3\% dos entrevistados com predominância entre as mulheres $(60,3 \% v s$. 39,7\%), superior à taxa global de infecção pelo HPV de pessoas que procuram serviços de DST no Brasil, $(41,2 \%)$ 31. Uma questão importante que deve ser considerada é que, neste trabalho, só foram incluídas as pessoas que tinham diagnóstico clínico e/ou etiológico de DST.

O longo período de incubação de algumas DST pode minimizar o problema da violência por parceiro íntimo após o diagnóstico. As pessoas com mais de um parceiro sexual na vida e que tinham verruga genital, praticaram menos violência por parceiro íntimo. A possibilidade da infecção ter sido contraída em uma relação anterior deve ser colocada pelo profissional de saúde, já que isto pode atenuar os conflitos gerados pela revelação do diagnóstico ao parceiro.

Vale destacar também que todas as pessoas que acreditavam ter contraído a DST por outro meio que não a relação sexual, não praticaram nenhum ato violento contra o seu parceiro. Contudo, não se justifica que profissionais de saúde forneçam informações infundadas sobre as formas de contaminação, por não saberem lidar com os conflitos gerados na relação, situação não rara no atendimento a pessoas com DST 32 . Somente estando informado sobre as formas de contaminação e prevenção, o individuo pode perceber a relação entre o seu comportamento, a doença e medidas eficazes de proteção.

Os dados deste trabalho devem ser interpretados com cautela devido a algumas limitações. Apesar de todos os cuidados com a coleta dos dados, como se trata de DST e violência, dois temas delicados e de difícil abordagem, os entrevistados podem ter omitido algumas informações.

A generalização dos resultados é de alcance limitado, representado as pessoas com DST que procuram atendimento em serviços de saúde, não sendo representativa de toda a população com DST da cidade de Fortaleza. Contudo, esses resultados destacam a importância de se considerar o problema da violência como um dos aspectos que pode dificultar o tratamento dos parceiros sexuais de portadores de DST. Por outro lado, pode contribuir para repensar as estratégias para a convocação desses parceiros e consequentemente o controle dessas patologias.

Os resultados deste estudo chamam a atenção dos profissionais que atendem pessoas com DST para que, durante o aconselhamento, iden- 
tifiquem as dificuldades vivenciadas diante da necessidade de revelação do diagnóstico, se colocando inclusive à disposição para contribuir na revelação. Faz-se necessário que profissionais e gestores estejam sensibilizados e comprome-

\section{Colaboradores}

M. A. L. Araújo e R. F. V. Andrade participaram da concepção do artigo, de todas as etapas da produção e foram responsáveis pela versão final. M. I. C. Dourado, A. B. E. Miranda e C. B. S. Reis participaram da revisão crítica e da aprovação da versão final. tidos com a causa, para que seja possível traçar estratégias viáveis para enfrentar a violência por parceiro íntimo diante da revelação do diagnóstico de DST.

\section{Referências}

1. Krug EG, Dahlberg LL, Mercy JA, Zwi AB, Lozano R, editors. World report on violence and health. Geneva: World Health Organization; 2002.

2. Mittal M, Senn TE, Carey MP. Mediators of the relation between partner violence and sexual risk behavior among women attending a sexually transmitted disease clinic. Sex Transm Dis 2011; 38: 510-5.

3. Senn TE, Carey MP, Vanable PA. The intersection of violence, substance use, depression, and STDs: testing of a syndemic pattern among patients attending an urban STD clinic. J Natl Med Assoc 2010; 102:614-20.

4. Barros C, Schraiber LB, França-Junior I. Associação entre violência por parceiro íntimo contra a mulher e infecção por HIV. Rev Saúde Pública 2011; 45:365-72.

5. Schraiber LB, D’Oliveira AFPL, França Júnior I; Grupo de Estudos em População, Sexualidade e Aids. Violência sexual por parceiro íntimo entre homens e mulheres no Brasil urbano, 2005. Rev Saúde Pública 2008; 42:127-37.
6. Decker MR, Miller E, McCauley HL, Tancredi DJ, Levenson RR, Waldman J, et al. Intimate partner violence and partner notification of sexually transmitted infections among adolescent and young adult family planning clinic patients. Int J STD AIDS 2011; 22:345-7.

7. Lövestad S, Krantz G. Men's and women's exposure and perpetration of partner violence: an epidemiological study from Sweden. BMC Public Health 2012; 12:945.

8. Parente EO, Nascimento RO, Vieira LJES. Enfrentamento da violência doméstica por um grupo de mulheres após a denúncia. Revista Estudos Feministas 2009; 17:445-65.

9. Guedes RN, Silva ATMC, Fonseca RMGS. A violência de gênero e o processo saúde-doença das mulheres. Esc Anna Nery Rev Enferm 2009; 13:625-31.

10. De Ferrante FG, Santos MA, Vieira EM. Violência contra a mulher: percepção dos médicos das unidades básicas de saúde da cidade de Ribeirão Preto, São Paulo. Interface Comun Saúde Educ 2009; 13:287-99. 
11. Vieira EM, Perdona GCS, Almeida AM, Nakano MAS, Santos MA, Daltoso D, et al. Conhecimento e atitude dos profissionais de saúde em relação à violência de gênero. Rev Bras Epidemiol 2009; 12:566-77.

12. World Health Organization. The global elimination of congenital syphilis: rationale and strategy for action. Geneva: World Health Organization; 2007.

13. Schraiber LB, Latorre MRD, França-Junior I, Segri NJ, D'Oliveira AFPL. Validade do instrumento WHO VAW STUDY para estimar violência de gênero contra a mulher. Rev Saúde Pública 2010; 44:658-66.

14. Marconi MA, Lakatos EM. Técnicas de pesquisas. 6a Ed. São Paulo: Editora Atlas; 2006.

15. Zaleski M, Pinsky I, Laranjeira R, Ramisetty-Mikler S, Caetano R. Violência entre parceiros íntimos e consumo de álcool. Rev Saúde Pública 2010; 44: 53-9.

16. Secretaria Especial de Políticas para as Mulheres, Presidência da República. Lei Maria da Penha. Lei no 11.340, de 7 de agosto de 2006: coíbe a violência doméstica e familiar contra a mulher. Brasília: Secretaria Especial de Políticas para as Mulheres, Presidência da República; 2011.

17. Tumwesigye NM, Kyomuhendo, GB, Greenfield TK, Wanyenze RK. Problem drinking and physical intimate partner violence against women: evidence from a national survey in Uganda. BMC Public Health 2012; 12:399.

18. Fonseca AM, Fernandes GJC, Tondowski CS, Noto AR. Padrões de violência domiciliar associada ao uso de álcool no Brasil. Rev Saúde Pública 2009; 43:743-9.

19. Leonard KE. Alcohol and intimate partner violence: when can we say that heavy drinking is a contributing cause of violence? Addiction 2005; 100:422-5.

20. Ray LA, Mackillop J, Leventhal A, Hutchison KE. Catching the alcohol buzz: an examination of the latent factor structure of subjective intoxication. Alcohol Clin Exp Res 2009; 33:2154-61.

21. Frye V, Ompad D, Chan C, Koblin B, Galea S, Vlahov $\mathrm{D}$. Intimate partner violence perpetration and condom use-related factors: associations with heterosexual men's consistent condom use. AIDS Behav 2011; 15:153-62.

22. Silverman JG, McCauley HL, Decker MR, Miller E, Reed E, Raj A. Coercive forms of sexual risk and associated violence perpetrated by male partners of female adolescents. Perspect Sex Reprod Health 2011; 43:60-5
23. Hess KL, Javanbakht M, Brown JM, Weiss RE, Hsu P, Gorbach PM. Intimate partner violence and sexually transmitted infections among young adult women. Sex Transm Dis 2012; 39:266-71.

24. Dude AM. Spousal intimate partner violence is associated with HIV and other STIs among married Rwandan women. AIDS Behav 2011; 15:142-52.

25. Frank S, Coelho EBS, Boing AF. Perfil dos estudos sobre violência contra a mulher por parceiro íntimo: 2003 a 2007. Rev Panam Salud Pública 2010; 27:376-81.

26. O’Donnell L, Agronick G, Duran R, Myint-U A, Stueve A. Intimate partner violence among economically disadvantaged young adult women: associations with adolescent risk-taking and pregnancy experiences. Perspect Sex Reprod Health 2009; 41:84-91.

27. Alam N, Streatfield PK, Shahidullah M, Mitra D, Vermund SH, Kristensen S. Effect of single session counseling on partner referral for sexually transmitted infections management in Bangladesh. Sex Transm Infect 2011; 87:46-51.

28. Whitaker DJ, Haileyesus T, Swahn M, Saltzman LS. Differences in frequency of violence and reported injury between relationships with reciprocal and nonreciprocal intimate partner violence. Am J Public Health 2007; 97:941-7.

29. Rutledge SE. Formation of personal HIV disclosure policies among HIV-positive men who have sex with men. AIDS Patient Care STDs 2009; 23:521-43.

30. Miranda MPM, Paula CS, Bordin IA. Violência conjugal física contra a mulher na vida: prevalência e impacto imediato na saúde, trabalho e família. Rev Panam Salud Pública 2010; 27:300-8.

31. Programa Nacional de DST e Aids, Secretaria de Vigilância em Saúde, Ministério da Saúde. Prevalências e frequências relativas de doenças sexualmente transmissíveis (DST) em populações selecionadas de seis capitais brasileiras, 2005. Brasília: Ministério da Saúde; 2008.

32. Araujo MAL, Silveira CB. Vivências de mulheres com diagnóstico de doença sexualmente transmissível - DST. Esc Anna Nery Rev Enferm 2007; 11:479-86. 


\section{Abstract}

The objective of this study was to investigate factors associated with intimate partner violence after disclosing the diagnosis of sexually transmissible diseases (STDs) in Fortaleza, Ceará State, Brazil. This cross-sectional study enrolled 221 individuals treated at STD clinics. Multivariate logistic regression was performed. A total of $28.1 \%$ of individuals committed some type of intimate partner violence after disclosure of the diagnosis. Committing intimate partner violence was associated with alcohol use $(O R=2.79$; 95\%CI: 1.25-6.22; $p=0.012$ ), the partner having relations with someone else during the current relationship $(O R=4.71 ; 95 \% C I$ : 2.24-9.91; $p=0.000)$, a history of violence prior to the STD (OR = 2.87; 95\%CI: 1.22-6.73; $p=0.015)$, and having suffered violence after diagnosis of the STD $(O R=$ 6.53; 95\%CI: 3.06-13.93; $p=0.000$ ). Intimate partner violence following disclosure of the STD signals that health professionals should identify patients' difficulties in revealing an STD diagnosis to their partners.

Sexually Transmitted Diseases; Intimate Partner Violence; Diagnosis; Violence

\section{Resumen}

El objetivo del estudio es investigar los factores asociados a la violencia por parejas sentimentales perpetrados tras la revelación del diagnóstico de enfermedades de tranmisión sexual (ETS), en Fortaleza, Ceará, Brasil. Estudio transversal realizado con 221 personas atendidas en servicios de referencia para DST. Se usó la razón de oportunidad con un intervalo de confianza de un 95\%. Practicaron algún tipo de violencia por parejas sentimentales tras la revelación del diagnóstico, 28, $1 \%$ de las personas. Practicar violencia por parejas sentimentales presentó asociación con el uso de alcohol (OR: 2,79; IC95\%: 1,25-6,22; $p=0,012)$, que el compañero se hubiera relacionado con otra persona durante la relación actual (OR: 4,71; IC95\%: 2,24-9,91; $p=0,000)$, haber cometido violencia anterior a ETS (OR: 2,87; IC95\%: 1,22-6,73; $p=0,015$ ) y haber sufrido violencia tras el diagnóstico de ETS (OR: 6,53; IC95\%: 3,06-13,93; $p=0,000$ ). La violencia por parejas sentimentales tras la revelación del diagnóstico de ETS indica que profesionales que atienden esa demanda, deben identificar las dificultades enfrentadas por el paciente al revelar el diagnóstico al compañero.

Enfermedades de Transmisión Sexual; Violencia de Pareja; Diagnóstico; Violencia
Recebido em 24/Jan/2015

Versão final reapresentada em 10/Set/2015 Aprovado em 22/Fev/2016 